\title{
Caracterización mecánica de aleaciones porosas, base Ti, producidas mediante la técnica de sinterización con espaciador
}

\author{
C. Tojal*, J. Devaud*, V. Amigó* y J.A. Calero**
}

\begin{abstract}
Resumen
La búsqueda de materiales adecuados para su uso como implante implica una mayor investigación sobre los biomateriales, como lo son el titanio y sus aleaciones. Respecto a sus propiedades mecánicas, se debe garantizar una resistencia mecánica suficiente como para soportar cargas en uso, al igual que su rigidez ha de ser parecida a la del hueso humano. En el presente trabajo se miden diferentes propiedades mecánicas de materiales porosos de titanio y de la aleación Ti6Al4V, producidos por vía pulvimetalúrgica mediante sinterización con espaciador. Los resultados muestran la relación entre la porosidad y las propiedades mecánicas, indicando los casos en los que se presenta un compromiso entre la rigidez y la resistencia mecánica.
\end{abstract}

\section{Mechanical characterization of porous Ti base alloys produced by sintering space-holder method}

\begin{abstract}
The search of suitable materials for use as an implant involves more research of biomaterials, like titanium and its alloys. Regarding their mechanical properties, it must be guaranteed mechanical strength to support loads in use, as well as its stiffness must be similar to the bone. In this paper it have been measured several mechanical properties of porous titanium and Ti6A14V alloy, produced by sintering powder metallurgy with space-holder method. The results show the relationship between porosity and mechanical properties and it is indicated in which cases it is presented a compromise between the stiffness and mechanical strength.
\end{abstract}

Keywords

Ti; Ti6Al4V; Space-holder sintering; Stiffness; Mechanical strength.

\section{INTRODUCCIÓN}

El campo de los biomateriales se ha convertido en un área vital para aumentar la calidad y longevidad de la vida humana. La búsqueda de materiales adecuados para su uso como implante implica una mayor investigación, siempre con el fin de encontrar el compromiso entre sus diferentes propiedades finales, como son la resistencia a la corrosión, las propiedades mecánicas o la facilidad de osteointegración, entre otras ${ }^{[1]}$.

Los implantes óseos corticales, los cuales están referidos a la sustitución de los principales huesos corticales (fémur, tibia, radio y húmero), además de los requisitos de biocompatibilidad, han de garantizar propiedades mecánicas adecuadas ${ }^{[2}$ y 3$]$ : La resistencia de los implantes ha de ser suficiente para soportar las cargas a las que se verá sometido el implante, con el fin de regenerar un hueso sano y fuerte. Por otro lado, también, resulta necesario estimular los osteoblastos o células de producción del nuevo hueso, mediante la transmisión eficiente de esfuerzos en la intercara entre el implante y el hueso ${ }^{[4}$ y 5$]$. Para ello, es fundamental que la rigidez del implante sea lo más semejante posible a la del hueso. El hecho de que la transmisión de esfuerzos en la intercara entre el implante y el hueso sea eficiente, acelera el tiempo de recuperación del paciente, evitando a la larga problemas de debilitamiento y aflojamiento[6 y 7].

Uno de los biomateriales más conocidos es el titanio, caracterizado, además de por su biocompatibilidad, por su bajo peso específico ${ }^{[8]}$. Frente a otros materiales metálicos utilizados como implantes, tales como el acero inoxidable y las aleaciones de cobalto-cromo, el titanio aporta una mejora de propiedades, como

\footnotetext{
* Instituto de Tecnología de Materiales, Universitat Politècnica de València, Camino de Vera s/n, 46022 Valencia, España. vamigo@mcm.upv.es.

${ }^{* *}$ Aleaciones Metálicas Sinterizadas (AMES), Ctra. Laureà Miró 388, 08980 Sant Feliu de Llobregat, Barcelona. jacalero@ames.es.
} 
son elevada resistencia específica y a fatiga, un módulo elástico más semejante al del hueso humano ${ }^{[9]}$ así como una excelente resistencia a la corrosión al contacto, tanto con el hueso como con los fluidos del cuerpo humano ${ }^{[10]}$.

Las aleaciones de titanio usadas como biomateriales se fabrican con objeto de mejorar las propiedades mecánicas del material puro. Tal es el caso de la conocida aleación Ti6Al4V, que presenta buena resistencia mecánica y elevada plasticidad ${ }^{[11}$ y 12$]$.

Aunque el valor de la rigidez del titanio y de la aleación Ti6Al4V de colada es más próximo al del hueso humano que el de otros metales, todavía, presenta un valor muy superior al mismo, en torno a 5 o 6 veces mayor ${ }^{[13}$ y 14]. Por ello, resulta de interés encontrar soluciones para reducir esta diferencia. Una de estas soluciones es la fabricación de materiales porosos de titanio que, por un lado, tienen menor rigidez y, por otro, con el uso de estos materiales se mejora la osteointegración debido a las características superficiales del implante ${ }^{[9]}$. Como elemento contraproducente, todas las propiedades mecánicas se ven mermadas por la aparición de porosidad, incluida la resistencia mecánica, propiedad necesaria para que el implante soporte cargas en uso ${ }^{[15]}$.

Mediante la técnica de sinterización de materiales particulados en presencia de espaciadores se pueden lograr materiales altamente porosos. El proceso se inicia con la mezcla del material base con el agente que funcionará como espaciador, seguido de una compactación. Posteriormente, el espaciador es eliminado a baja temperatura y, finalmente, la pieza es sinterizada a elevada temperatura, lo que confiere las propiedades finales al material. Como espaciador se han utilizado diversos materiales entre los que está el bicarbonato de amonio, el cual se elimina fácilmente por descomposición térmica. El método de espaciadores es una de las formas más fáciles de obtener piezas porosas de titanio ${ }^{[9]}$, cuyo control de forma, tamaño y cantidad de poros viene determinado por la geometría, dimensión y volumen de espaciador.

En el presente trabajo se estudian las propiedades mecánicas de rigidez y resistencia de materiales porosos de titanio y la aleación Ti6Al4V producidos por vía pulvimetalúrgica mediante sinterización con espaciador. La caracterización mecánica se ha llevado a cabo con medida de microdureza y ensayos de flexión, compresión y ultrasonidos. La caracterización microestructural se ha realizado con microscopía óptica y electrónica. El objetivo principal es relacionar la porosidad con las propiedades mecánicas, al igual que garantizar un compromiso entre la rigidez y la resistencia mecánica, de los materiales obtenidos.

\section{MATERIALES $Y$ PROCEDIMIENTO EXPERIMENTAL}

Los polvos utilizados para la investigación fueron polvo de titanio y del prealeado Ti6Al4V, comercializados por Se-Jong Materials Co. LTD, cuyas composiciones se indican en la tabla I. El fabricante indica un tamaño de partícula (-325) mesh, aunque para esta investigación se utilizó la fracción de polvo de tamaño inferior a $25 \mu \mathrm{m}$.

Como espaciador se utilizó el bicarbonato de amonio, comercializado por la casa Alfa Aesar $®$, con una pureza del $99.9 \%$. En vista de anteriores estudios y con objeto de conseguir mejores propiedades finales ${ }^{[16]}$, el espaciador fue tamizado para conseguir granulometrías entre 425 y $600 \mu \mathrm{m}$.

Además de trabajar con cada material sin adicionar espaciador, se realizaron mezclas elementales con 60, 70 y $80 \%$ vol. de bicarbonato de amonio y, posteriormente, se obtuvieron compactos a $400 \mathrm{MPa}$ de presión de compactación. Para cada ensayo se prepararon 3 probetas y se midieron todas las propiedades a las mismas.

Las matrices de compactación utilizadas fueron las siguientes: para los ensayos de flexión se usó una matriz rectangular de dimensiones $12 \times 30 \mathrm{~mm}^{2}$; pra los ensayos de compresión se usó una matriz cilíndrica de 10 mm de diámetro. La eliminación del bicarbonato tuvo lugar en estufa,a $100^{\circ} \mathrm{C}$ durante 10 h. La sinterización se realizó en vacío en un horno Carbolite modelo HVT $15 / 75 / 450$, a la temperatura de $1.50^{\circ} \mathrm{C}$ durante $2 \mathrm{~h}$, tilizando velocidades de calentamiento entre $10 \mathrm{y}$ $15^{\circ} \mathrm{C} / \mathrm{min}$. El enfriamiento de las muestras se realizó en atmósfera de argón, igualmente a bajas velocidades para no dañar los elementos del horno.

La densidad de las muestras sinterizadas se midió mediante el método de Arquímedes según la norma

Tabla I. Composición química de los polvos utilizados

Table I. Chemical composition of powders

$$
\text { Ti Ti6Al4V }
$$

\begin{tabular}{cccr}
\hline Componente & \% peso & Componente & \multicolumn{1}{c}{ \% peso } \\
\hline $\mathrm{Ti}$ & 99,7 & $\mathrm{Ti}$ & 88,83 \\
$\mathrm{Mg}$ & 0,009 & $\mathrm{Al}$ & 6,62 \\
$\mathrm{Si}$ & 0,09 & $\mathrm{~V}$ & 4,55 \\
$\mathrm{Mn}$ & 0,006 & $\mathrm{Fe}$ & 0,02 \\
$\mathrm{Fe}$ & 0,029 & $\mathrm{Zr}$ & 0,03 \\
$\mathrm{O}$ & 0,362 & $\mathrm{O}$ & Máx. 0,55 \\
$\mathrm{N}$ & 0,01 & $\mathrm{~N}$ & Máx. 0,5 \\
$\mathrm{H}$ & 0,013 & $\mathrm{H}$ & Máx. 0,3 \\
\hline
\end{tabular}


ASTM C 373 -88, determinándose la porosidad total de las piezas.

La preparación metalográfica ha sido realizada siguiendo las técnicas convencionales. Las muestras sinterizadas fueron caracterizadas por microscopía óptica con un microscopio Nikon Microphot FX y por microscopía electrónica con un microscopio JEOL JSM 6400.

La microdureza, medida según el ensayo de dureza Vickers (HV), se ha obtenido con un microdurómetro Matsuzawa MHT2, aplicando una carga de $200 \mathrm{~g}$ durante 15 s y utilizando un penetrador Vickers.

Los ensayos de flexión y compresión se realizaron con una prensa Instron modelo 4204 y células de carga de 5 o $50 \mathrm{KN}$, según la necesidad en cada ensayo. Del ensayo de flexión de obtuvieron las curvas de cada material, además de la resistencia máxima y la pendiente de la curva en la zona elástica o módulo, el cual se ha relacionado con el módulo de los materiales de chapa correspondientes (21 GPa para Ti y $29 \mathrm{GPa}$ para Ti6Al4V). Del ensayo de compresión se obtuvieron las curvas y la resistencia máxima de carga o de rotura.

Con un equipo de ultrasonidos Krautkramer USM 35 se midió la rigidez del material, la cual se ha comparado con la rigidez obtenida para los materiales de colada correspondientes (105 GPa para Ti y 110 GPa para Ti6Al4V).

\section{RESULTADOS Y DISCUSIÓN}

\subsection{Porosidad}

El dato de porosidad de las muestras de titanio, en función de la cantidad de espaciador, se observa en la figura 1. Los resultados expuestos son datos medios de varios

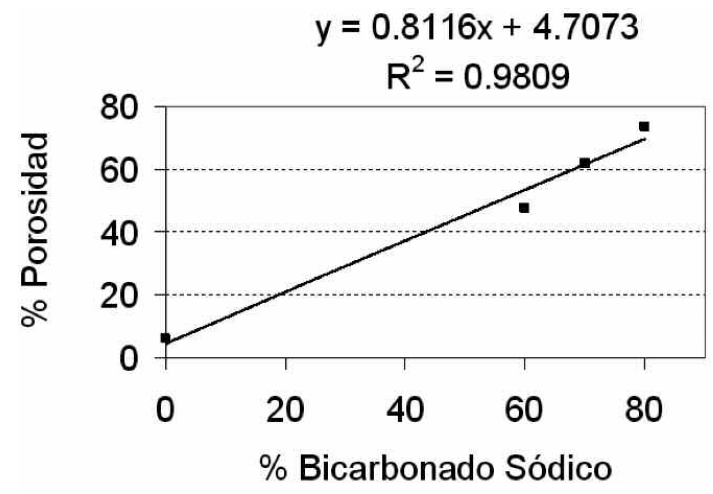

Figura 1. Relación entre porosidad y cantidad de espaciador para probetas de titanio.

Figure 1. Relation between porosity and spacer amount for titanium samples. valores, encontrándose una dispersión inferior al $5 \%$ con respecto a la media. Existe una correlación lineal positiva fuerte entre ambas variables (regresión lineal cercana a 1) lo que indica una fuerte dependencia entre la porosidad final y el porcentaje en volumen de espaciador utilizado en la mezcla, al menos comprobado cuando se trabaja con materiales con porosidades superiores al $50 \%$. Iguales resultados se obtienen cuando el material base es la aleación Ti6Al4V, tal como se puede observar en la figura 2. Esto indica que el valor de porosidad es un valor fácilmente controlable en las investigaciones, al poder controlar la cantidad de espaciador utilizada durante la preparación de las mezclas. La porosidad es un valor que influye notablemente en las propiedades mecánicas ${ }^{[17]}$ por lo que, si se controla, se pueden llegar a controlar las propiedades mecánicas finales de los materiales.

En las micrografías de la figura 3 se observa la presencia de poros y su morfología. Aunque existe cierta heterogeneidad en el tamaño de poros, se observa, en muchos, que su tamaño coincide con el tamaño de las partículas de espaciador utilizadas, si bien la cantidad de porosidad visible en las muestras aumenta con el aumento de la cantidad de espaciador en las mezclas. Por ello, muestras sinterizadas con un $80 \%$ vol. de espaciador presentan muy elevada porción de porosidad y, por tanto, poros de gran tamaño, como consecuencia del solapamiento entre partículas de espaciador.

\subsection{Propiedades mecánicas}

Entre las diferentes propiedades mecánicas de los materiales, se ha medido la microdureza. El tamaño medio de las huellas obtenidas se encuentra en torno a 1.100. $1.500 \mathrm{~mm}^{2}$. La tabla con los resultados obtenidos

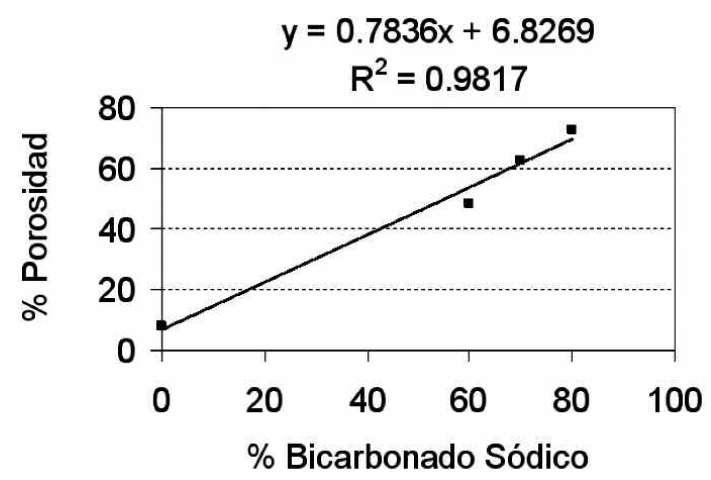

Figura 2. Relación entre porosidad y cantidad de espaciador para probetas de Ti6Al4V.

Figure 2. Relation between porosity and spacer amount for Ti6Al4V samples. 

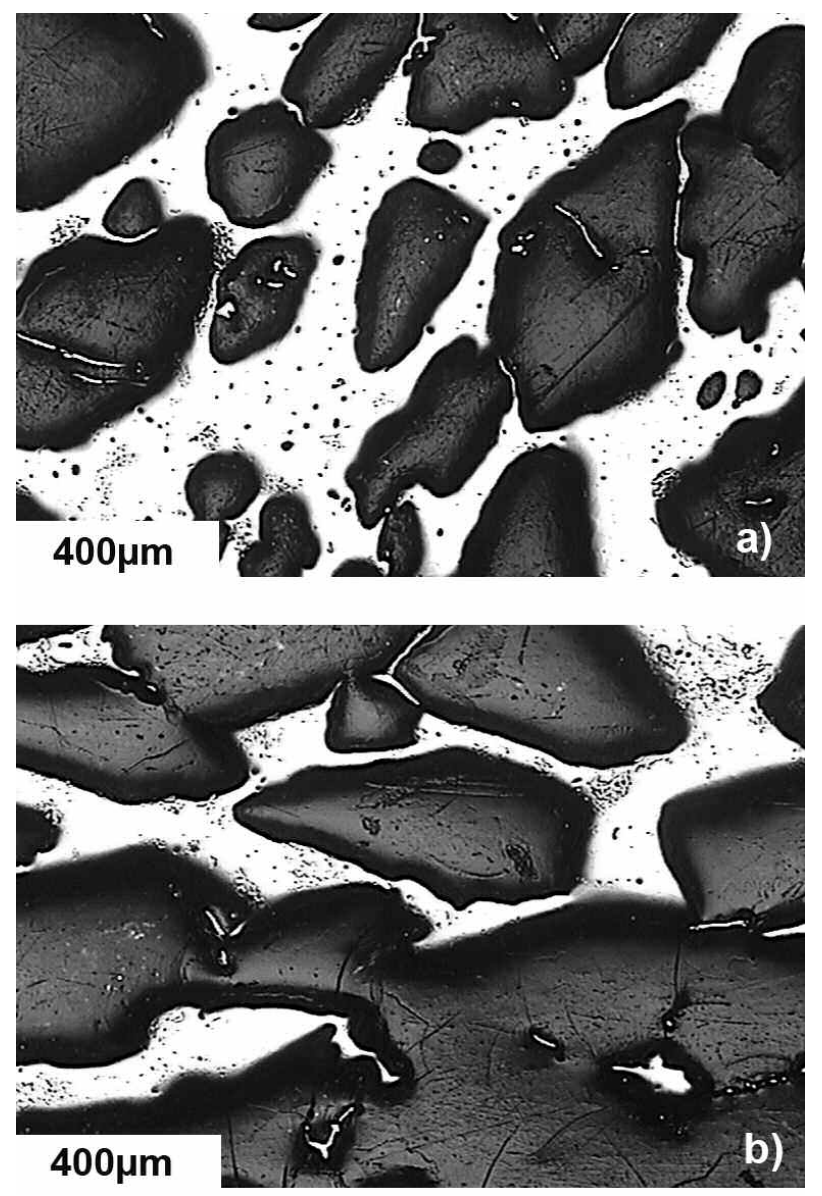

Figura 3. Micrografías de muestras de Ti6Al4V con espaciador: a) $60 \%$ vol. - b) $80 \%$ vol.

Figure 3. Micrographs of Ti6Al4V samples with spacer: a) $60 \%$ vol. - b) $80 \%$ vol.

(Tabla II) muestra valores similares para todos los casos, en torno a $330 \mathrm{HV}$ para las muestras de titanio y 304 HV para las muestras de la aleación Ti6Al4V, por lo que esta no es una propiedad notablemente afectada

Tabla II. Microdurezas de los materiales estudiados

Table II. Microhardness of investigated materials

\begin{tabular}{ccc}
\hline \multirow{2}{*}{$\begin{array}{c}\text { \% Bicarbonato } \\
\text { sódico }\end{array}$} & Ti & Ti6Al4V \\
\cline { 2 - 3 } & \multicolumn{2}{c}{ HV } \\
\hline 0 & $327 \pm 35$ & $308 \pm 25$ \\
60 & $331 \pm 31$ & $301 \pm 19$ \\
70 & $334 \pm 31$ & $303 \pm 32$ \\
80 & $328 \pm 27$ & $304 \pm 23$ \\
\hline
\end{tabular}

por la porosidad final del material inducida por la mezcla con espaciador y, más bien, dependerá de las condiciones de sinterización como temperatura y tiempo.

Los resultados de compresión y flexión aportan la carga máxima que soporta cada probeta ensayada antes de su rotura o inicio de grietas. Los resultados obtenidos para las muestras de titanio y la aleación Ti6Al4V se observan en las tablas III y IV, respectivamente. El valor de resistencia disminuye a medida que aumenta la cantidad de espaciador y, por lo tanto, la porosidad. Aunque se observan mayores resistencias para la aleación Ti6Al4V que para las muestras de titanio, la tendencia para ambos materiales resulta similar.

La disminución de resistencia mecánica se hace más notable en el ensayo de flexión, pasando a un 17-15 \% de la resistencia, de la muestra sinterizada sin espaciador a la sinterizada con un $60 \%$ vol. de espaciador. Para muestras con mayor porosidad, la reducción de resistencia es notable situándose, en todos los casos, en valores menores del $5 \%$ de la resistencia del material sinterizado sin espaciador. La resistencia empieza a depender más de la porosidad que del material de partida, cuanto mayor es la cantidad de porosidad inducida.

Tabla III. Resistencia a compresión y flexión para probetas de titanio

Table III. Compression and bending resistance of titanium samples

\begin{tabular}{|c|c|c|c|}
\hline \multirow{2}{*}{$\begin{array}{c}\% \text { Bicarbonato } \\
\text { sódico }\end{array}$} & \multicolumn{3}{|c|}{$\mathrm{Ti}$} \\
\hline & $\sigma_{\mathrm{C}}(\mathrm{MPa})$ & $\sigma_{\mathrm{F}}(\mathrm{Mpa})$ & $\sigma_{F}$ rel. (\%) \\
\hline 0 & $>2.500$ & $1.432 \pm 60$ & 100 \\
\hline 60 & $670 \pm 180$ & $249 \pm 20$ & 17 \\
\hline 70 & $286 \pm 24$ & $133 \pm 12$ & 9 \\
\hline 80 & $116 \pm 14$ & $57 \pm 8$ & 4 \\
\hline
\end{tabular}

Tabla IV. Resistencia a compresión y flexión para probetas de Ti6AI4V

\section{Table IV. Compression and bending resistance of Ti6Al4V samples}

\begin{tabular}{|c|c|c|c|}
\hline \multirow{2}{*}{$\begin{array}{c}\% \text { Bicarbonato } \\
\text { sódico }\end{array}$} & \multicolumn{3}{|c|}{ Ti6Al4V } \\
\hline & $\sigma_{C}(\mathrm{MPa})$ & $\sigma_{\mathrm{F}}(\mathrm{Mpa})$ & $\sigma_{F}$ rel. $(\%)$ \\
\hline 0 & $>2.500$ & $1.866 \pm 80$ & 100 \\
\hline 60 & $848 \pm 127$ & $318 \pm 17$ & 15 \\
\hline 70 & $479 \pm 36$ & $143 \pm 19$ & 8 \\
\hline 80 & $136 \pm 18$ & $56 \pm 3$ & 3 \\
\hline
\end{tabular}


Curvas representativas obtenidas por el ensayo de flexión se observan, para las muestras de la aleación Ti6Al4V, en la figura 4. Similares resultados se obtuvieron para las muestras de titanio. La tendencia de las muestras, a medida que aumenta la porosidad, es la de soportar menos carga, al igual que un cambio en el módulo. Este cambio se puede observar en la figura 5, donde se aportan datos del módulo de flexión relativo al módulo de los materiales de chapa correspondientes, obtenidos al realizar el mismo ensayo de flexión. Los valores de rigidez, alcanzados para los materiales porosos, son considerablemente inferiores a la chapa e, incluso, a los mismos materiales sinterizados sin espaciador. Las imágenes de la fractura a flexión correspondientes a los materiales porosos de titanio se observan en la figura 6, donde se aprecia la alta porosidad y, relativamente, poca área superficial frágil que presentan los materiales. Los resultados que se obtienen con la aleación Ti6Al4V son similares.

Las curvas de compresión a modo de ejemplo para muestras de titanio se observan en la figura 7 . También, se obtuvieron similares esultados para muestras de la aleación Ti6Al4V. A medida que aumenta

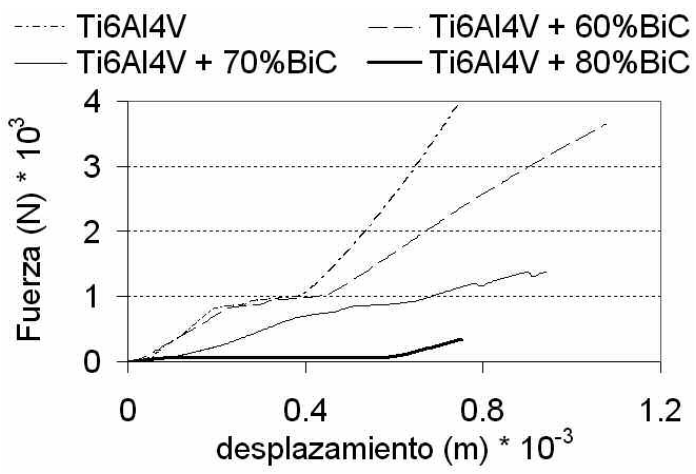

Figura 4. Curvas de flexión para muestras de Ti6Al4V.

Figure 4. Bending curves for Ti6Al4V samples.

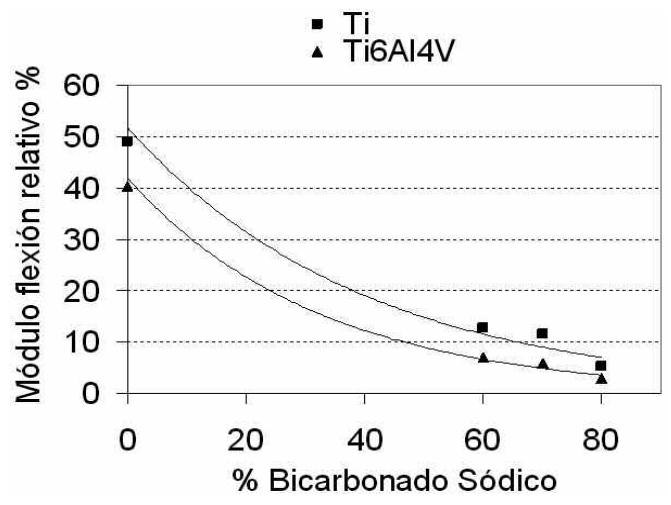

Figura 5. Módulo de flexión relativo en función de $\%$ vol. de espaciador.

\section{Figure 5. Relative bending modulus related to} $\%$ vol. spacer.

la porosidad, la probeta soporta cargas inferiores, presentando una ductilidad elevada, en todos los casos. Observaciones semejantes han sido realizadas por otros autores $^{[18]}$.

Mediante ultrasonidos se ha medido la rigidez de las muestras, la cual se compara con la rigidez de los correspondientes materiales de colada, en la figura 8 . Se observa que la rigidez de los materiales porosos desarrollados es inferior a $20 \mathrm{GPa}$, constituyendo un valor más parecido a los del hueso humano. A la vista de los resultados, la rigidez y la porosidad se muestran relacionados de forma inversamente proporcional, correlacionándose con el modelo predicho por Gibson y Ashby ${ }^{[19]}$ : Estos autores proponen un modelo de correlación para espumas de célula abierta con densidad relativa inferior a 0,2, según el cual algunas propiedades físicas y mecánicas siguen la ley expresada en la ecuación (1):

$$
A\left(\rho_{r}\right)=A_{0} \times r_{r}^{n}
$$

donde, $\rho_{r}$ es la densidad relativa y $\mathrm{A}_{0}$ y $n$ son parámetros del material y dependen de diversos factores, como
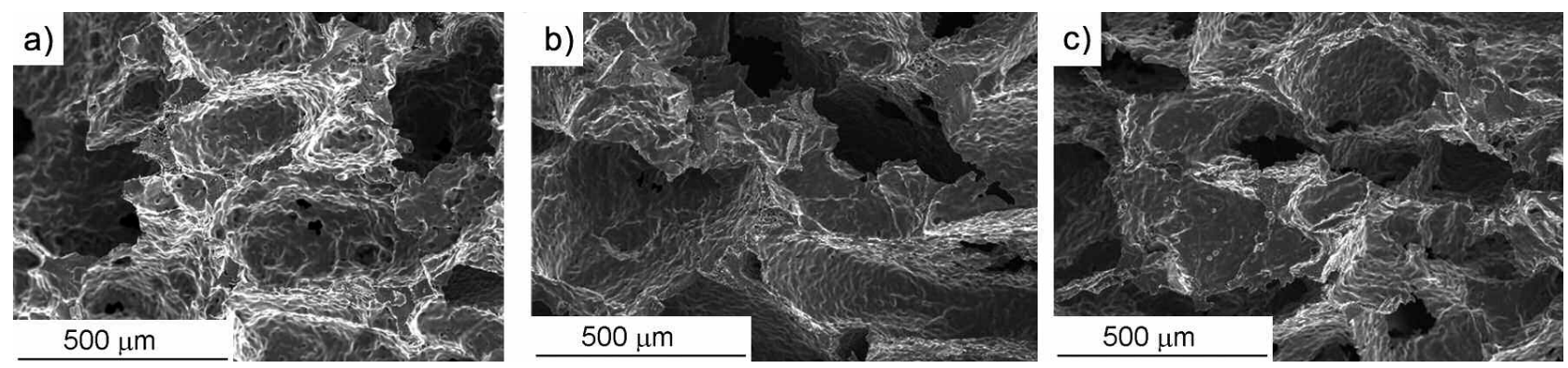

Figura 6. Micrografías de fractura de muestras de Ti con: a) $60 \%$, b) 70 y c) $80 \%$ vol. espaciador.

Figure 6. Fracture micrographs of Ti samples with: a) $60 \%$, b) $70 \%$ and c) $80 \%$ vol. spacer. 


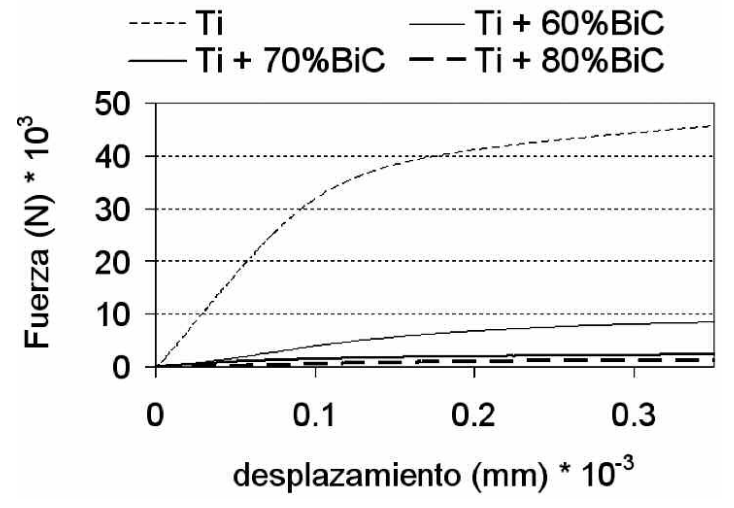

Figura 7. Curvas de compresión para muestras de titanio.

Figure 7. Compression curves for titanium samples.

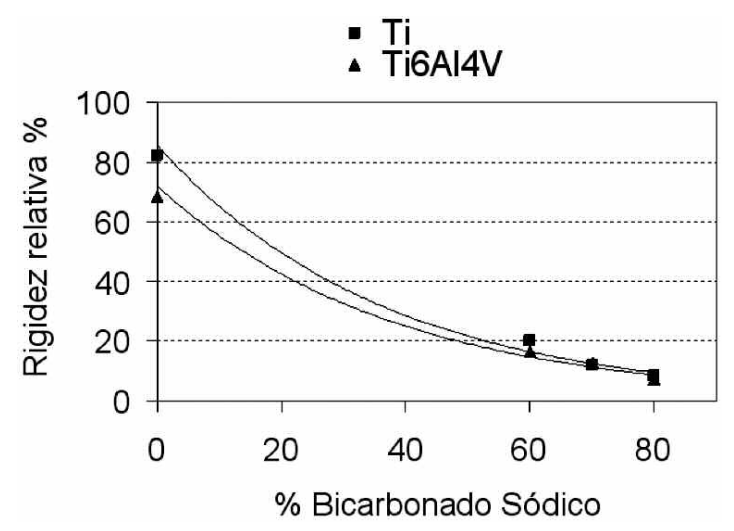

Figura 8. Rigidez relativa (ultrasonidos) en función de \% vol. de espaciador.

Figure 8. Relative stiffness (ultrasounds) related to \% vol. spacer.

la influencia de una membrana exterior densa o la dirección de espumación. Para espumas de mayor densidad, la extensión o compresión de las aristas de las celdillas modifican su curvatura por lo que, al variar el mecanismo de deformación, es de esperar una desviación respecto al modelo propuesto ${ }^{[20]}$. Este sería el caso de los materiales desarrollados en el presente trabajo; Como la densidad relativa se relaciona con la porosidad, $P$, por la ecuación (2), un aumento de porosidad implica una disminución de la densidad relativa.

$$
P=1-\rho_{r}
$$

Por lo tanto la densidad relativa para las muestras sinterizadas con espaciador presenta valores situados entre 0,5 y 0,3. La ecuación (1) se extiende a estos valores, hallando una relación inversamente proporcional entre la densidad relativa y las propiedades de rigidez y resistencia a compresión y flexión.

Una vez conocidas las propiedades mecánicas de los materiales desarrollados, conviene valorar la eficacia del método de procesamiento. En la tabla $\mathrm{V}$ se aportan datos de propiedades mecánicas de huesos corticales ${ }^{[6,}$ 13, 21 y 22]. Para los cuatro huesos corticales (fémur, tibia, radio y húmero) los datos aportados son los siguientes: La rigidez oscila entre 12 y $27.4 \mathrm{GPa}$; La resistencia a flexión se encuentra en torno a $100 \mathrm{MPa}$; La resistencia a compresión oscila entre 114 y $206 \mathrm{MPa}$ dependiendo del hueso ensayado. Por lo que para garantizar una buena integración del implante y un adecuado funcionamiento en uso, la rigidez de los materiales tiene que ser parecida a lo comentado, mientras que la resistencia a compresión ha de ser superior a $150 \mathrm{MPa}$ y la resistencia a flexión mayor de $100 \mathrm{MPa}$.

En la figura 9 se representan las tres propiedades mecánicas (rigidez medida por ultrasonidos, resistencia a compresión y resistencia a flexión), obtenidas para

Tabla V. Propiedades mecánicas de diferentes huesos corticales de cuerpo humano[7, 9, 17 y 18]

Table V. Mechanical properties of several cortical bones of human body[7, 9, 17 and 18]

\begin{tabular}{cccc}
\hline Hueso & $\mathbf{E}(\mathbf{G P a})$ & $\mathbf{s}_{\mathbf{C}}(\mathbf{M P a})$ & $\mathbf{s}_{\mathbf{F}}(\mathbf{M p a})$ \\
\hline Fémur & $12-27,4$ & $167-206$ & \\
Tibia & $14-18,1$ & $130-201$ & 100 \\
Radio & $12-18,6$ & $114-200$ & \\
Húmero & 17,2 & 132 & \\
\hline
\end{tabular}

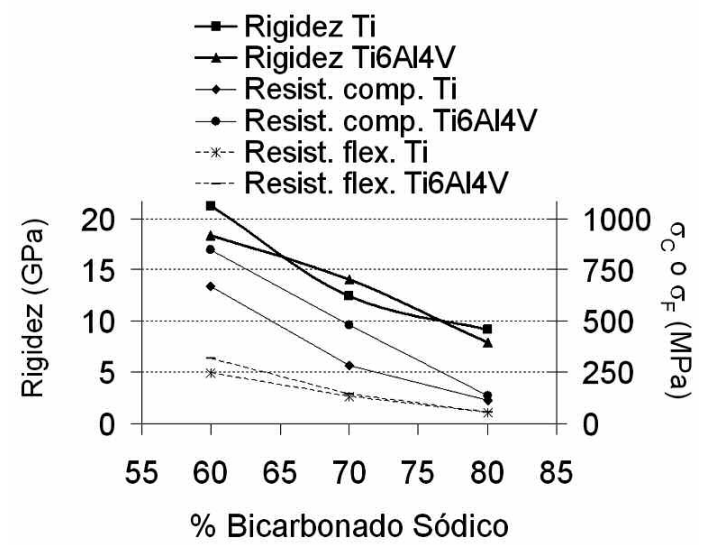

Figura 9. Propiedades mecánicas en función de $\%$ vol. de espaciador.

Figure 9. Mechanical properties related to \% vol. spacer. 
cada material, en función de la cantidad de espaciador utilizado. Se observan valores de estas propiedades que se acercan a lo buscado para un material de implante, si bien los materiales más porosos, con un $80 \%$ en volumen de espaciador, poseen valores de resistencia inferiores a lo buscado. Existe otro tipo de implantes óseos para huesos esponjosos que requieren un valor inferior de las propiedades comentadas, por lo que podría buscarse una nueva alternativa a la producción estos implantes, con espumas metálicas producidas por vía pulvimetalúrgica.

\section{CONCLUSIONES}

En el presente trabajo se han desarrollado materiales porosos de titanio y la aleación Ti6Al4V, mediante vía pulvimetalúrgica por sinterización con espaciador. Se ha encontrado una relación lineal entre la porosidad obtenida y la cantidad de espaciador utilizada para el procesado de los materiales, garantizada cuando la porosidad es superior al $50 \%$.

Las propiedades mecánicas de los materiales obtenidos se han medido con el fin de garantizar el uso de los mismos como implante. Se han utilizado ensayos de compresión y flexión, además de medir la microdureza de las muestras y la rigidez por ultrasonidos. Excepto para las microdurezas, las propiedades mecánicas obtenidas presentan una relación con la porosidad, siendo de menor magnitud cuanto mayor es la cantidad de porosidad del material.

La mayoría de los materiales procesados presentan propiedades mecánicas adecuadas para su uso como implante de huesos corticales. Las aleaciones más porosas no cumplen con las expectativas, aunque se podrían aplicar a implantes de huesos con menores exigencias mecánicas, como son los huesos esponjosos.

\section{Agradecimientos}

Los autores agradecen al área de Ciencia de Materiales e Ingeniería Metalúrgica de la Universidad de Sevilla la ayuda prestada en el cálculo de la rigidez por ultrasonidos. Igualmente, los autores desean agradecer la financiación obtenida por el Ministerio de Ciencia e Innovación (proyecto PET2008_0158_02) y a la beca Grisolia /2009/040.

\section{REFERENCIAS}

[1] B.D. Ratner, A.S. Hoffman, F.J. Schoen y J.E. Lemons, Biomaterials Science: An introduction to materials in medicine, Elsevier Academic Press, California, EE. UU., 2004, pp. 137-152.
[2] I.H. Oh, H. Segawa, N. Nomura y S. Hanada, Mater. Trans. 44 (2003) 657-660.

[3] C.F. Li, Z.G. Zhu y T. Liu, Powder Metall. 48 (2005) 237-240.

[4] S.J. Simske, R.A. Ayers y T.A. Bateman, Mater. Sci. Forum 250 (1997) 151-182.

[5] M. Vallet-Regí y L. Munuera, Biomateriales, aquí y ahora, Ed. Dykinson, Madrid, España, 2000, p. 267.

[6] M. Comín, J.L. Peris, J.M. Prat, J.R. Decoz, P.M. Vera y J.V. Hoyos, Biomecánica de la fractura ósea y técnicas de reparación, Ed. Publicaciones UPVIBV, Valencia, España, 1999, pp. 65-121.

[7] J.P. Li, S.H. Li, C.A. Van-Blitterswijk y K. DeGroot, J. Mater. Sci.: Materials in Medicine 17 (2006) 179-185.

[8] M. Geetha, A.K. Singh, R. Asokamani y A.K. Gogia, Prog. Mater. Sci. 54 (2009) 397-425.

[9] Z. Esen y S. Bor, Scr. Mater. 56 (2007) 341-344.

[10] N. Ibris y J.C. Mirza-Rosca, J. Electroanal. Chem. 526 (2002) 53-62.

[11] V. Amigó y A.V. Escuder, Materials per al disseny industrial. Aliatges metàl.lics, Ed. Publicaciones UPV, Valencia, España, 2003, pp. 176-182.

[12] C. Leyens y M. Peters, Titanium and Titanium Alloys. Fundamentals and Applications, Ed. Wiley VchGmbh\&Co, Weinheim, Alemania, 2003, p. 423.

[13] R.B. Heimann, CMU. Journal, 1 (2002) 23-46.

[14] ASM Handbook vol.9: Metallography and Microstructures, ASM International, Ohio, EE. UU., 2004, pp. 964-966.

[15] L.J.Gibson, Ann. Rev. Mater. Sci. 30 (2000) 191227.

[16] L. Reig, Tesis Doctoral, Instituto de Tecnología de Materiales, Universidad Politécnica de Valencia, 2010.

[17] Handbook of Non-Ferrous Metal Powders: Technologies and Applications, ASM Handbook, 2009, pp. 221-223.

[18] M. Bram, C. Stiller, H.P. Buchkremer, D. Stöver y H. Baur, Adv. Eng. Mater. 2 (2000) 196-199.

[19] L.J. Gibson y M.F. Ashby, Cellular Solids: Structure and Properties (Second edition), Cambridge University Press, Cambridge, Inglaterra, 1997, pp. 175-231.

[20] H.P. Degischer y B. Kriszt, Handbook of Cellular Metals: Production, Processing, Applications, Ed. Wiley VchGmbh\&Co, Weinheim, Alemania, 2002, pp. 179-190.

[21] C.E. Wen, Y. Yamada, K. Shimojima, Y. Chino, T. Asahina y M. Mabuchi, J. Mater. Sci.: Materials in Medicine 13 (2002) 397-401.

[22] K. Asaoka y M. Kon, Thermec'2003, pts 1-5, 4264, Madrid, España, 2003, pp. 3.079-3.084. 